\title{
Applying Machine Learning Techniques to improve Linux Process Scheduling
}

\author{
Atul Negi, Senior Member, IEEE, Kishore Kumar P. \\ Department of Computer and Information Sciences \\ University of Hyderabad \\ Hyderabad, INDIA 500046 \\ atulcs@uohyd.ernet.in, kishoregupta_os@yahoo.com
}

\begin{abstract}
In this work we use Machine Learning (ML) techniques to learn the CPU time-slice utilization behavior of known programs in a Linux system. Learning is done by an analysis of certain static and dynamic attributes of the processes while they are being run. Our objective was to discover the most important static and dynamic attributes of the processes that can help best in prediction of CPU burst times which minimize the process TaT (Turn-around-Time). In our experimentation we modify the Linux Kernel scheduler (version 2.4.20-8) to allow scheduling with customized time slices. The "Waikato Environment for Knowledge Analysis" (Weka), an open source machine-learning tool is used to find the most suitable ML method to characterize our programs. We experimentally find that the $C 4.5$ Decision Tree algorithm most effectively solved the problem. We find that predictive scheduling could reduce $\mathrm{TaT}$ in the range of $1.4 \%$ to $5.8 \%$. This was due to a reduction in the number of context switches needed to complete the process execution. We find our result interesting in the context that generally operating systems presently never make use of a program's previous execution history in their scheduling behavior.
\end{abstract}

Index Terms-Machine learning, Process Scheduling, turnaround-time.

\section{INTRODUCTION}

G ENERALLY process schedulers allocate CPU time slices to a process according to a scheduling algorithm that does not use any previous execution history of the process. It might be possible to better utilize resources if we could "recognize" a program and predict its resource requirements. For example, consider a process which was allocated a fixed CPU time is pre-empted while having a very small CPU time needed for completion. Such preemption, increases number of context switches (also called as a process switch or task switch), it causes invalidation of caches and pipelines, swapping of buffers and so on [6]. Thus ultimately this increases TaT of the program. (TaT is the delay between process submission and process completion). Thus we observe that by characterizing or recognizing programs it may be possible to understand their previous execution history and predict their resource requirements. In this paper we address the problem of, how to minimize the TaT of programs by using ML techniques. We discover certain static and dynamic characteristics of a program are taken as features which the machine learning techniques use to predict CPU burst times. We call Special Time Slice or STS as the CPU burst time that minimizes turnaround time.
The rest of this paper first discusses related work in Section II, an overview of machine learning techniques in Section III, we rerview Linux process scheduling in Section IV, and then describes our implementation in Section V. Section VI describes our experimentation. Section VII presents the conclusions and describes possible future work.

\section{RELATED WORK}

The work [10] by Suranauwarat and Taniguchi, presents an approach to remember the previous execution behavior of certain well-known programs. They study the process times of these programs in variousimilaritys states. The knowledge of the program flow sequence (PFS) is used to extend the $C P U$ time slice of a process. PFS of a process is computed from its past execution history. $P F S$ characterizes the process execution behavior and is used to decide whether the program executing currently needs additional time. They set a threshold $T_{m}$ called as maximum dispatch delay time, which determines the time limit for context switching; and is a multiple of the delay for minimum process switching time. They control the $C P U$ time of a process $T_{p}$, by either reducing or increasing some scaling feature. They conclude from experimental results that overall processing time is reduced for known programs. To schedule a process they search for its name in the PFS knowledge base and thus improve its behavior.

In the paper by Smith et al. [7], the authors predicted the application run times using historical information. They present a technique for deriving predictions for the run times of parallel applications from the run times of similar applications that have executed in the past. They use the following characteristics to define similarity: user, queue, load leveler script, arguments, network adapter, number of nodes, maximum run time, submission time, start time, run time. These characteristics are used to make a template which can find the similarity by matching. They use genetic algorithms for identifying "good templates" for a particular workload. A template defines the similarity between two applications. They use Genetic Algorithm (GA) techniques to determine those application characteristics that yield the best definition of similarity for making predictions. GAs are probabilistic techniques and are well known for exploring large search spaces. However from a machine learning perspective, GAs are expensive in terms of computation [15] and also their 
results are considered fragile.

In the paper by Gibbons [5], the authors used Statistical Regression methods for prediction. Wh ile regression methods work well on numeric data but cannot readily be used for nominal data. In the paper by Fredrik et al. [4], the authors described an application signature model used for predicting application performance on a given set of grid resources. In this model, they introduced the notion of application intrinsic behaviour to separate the performance effects of the runtime system from the behaviour inherent in the application itself.The signature model is used as the basis for performance predictions. So their approach combines the knowledge of application intrinsic behaviour with run-time predictions of resources. They also define application intrinsic metrics as metrics that are solely dependent on the application code and problem parameters.

From this brief review of related literature, we draw the following conclusions:

- It is possible to profitably predict scheduling behavior.

- However the success of the approach depends upon the ML technique used to train on previous programs execution behavior.

- More importantly for the ML techniques to succeed, it needs a suitable characterization of the program attributes (features) that are most significant for prediction.

\section{AN INTRODUCTION TO MACHINE LEARNING TECHNIQUES USED}

In this section we bring in some background information about the machine learning techniques which were used in the experimentation. The field of machine learning (ML) [15] is concerned with the question of how to construct computer programs that automatically improve with experience. ML methods are also similar to Pattern Recognition methods as [16]. The most important aspect is that while training or learning stage several attributes are used to describe the objects being studied. In our case the objects being studied are programs and we have come up with the best attributes that can desribe programs for prediction. [8]. However the success of a ML method also depends upon the classifiers used. Certain classifiers are more successful for different data or problem domains. Here we just give a cursosry introduction to the two most effective which were used [8]

The Learning Algorithms (Classifiers): For our experiments, we selected a representative set of standard machine learning algorithms with different model classes. All of these are available in the "Waikato Environment for Knowledge Analysis (Weka) [9] , an open source machine learning tool. The selected learner types (classifier types) were: "Trees and Lazy which are listed below:

- C4.5 (or J48 in Weka [9] ) is a Reduced-Pruned Decision Tree Learner. Decision Tree learning is one of the most widely used and practical methods for inductive inference. It is a method for approximating discrete-valued functions, and is robust to noisy data and capable of learning disjunctive expressions.
- IB $k$ Learner $(k-\mathrm{NN})$ : This is an instance based learner antd assumes all instances are points in an n-dimensional space. The nearest neighbors (nn) of an instance are defined in terms of the standard Euclidean Distance. The class label of a new instance is found from the $k$ instances nearest to it by assigning to it the majority label of the $k$-NN [15] [9].

\section{A REVIEW OF LINUX PROCESS SCHEDULING CONCEPTS}

In this section, we review the Linux process scheduling concepts which we use to develop the modified scheduler as described in Section V.

Scheduler: The idea behind a scheduler is simple. Its objective is to best utilize processor time. Assuming there are runnable processes, a process should always be running. If there are more processes than processors in a system, some processes will not always be running. These processes are waiting to run. Deciding which process runs next, given a set of runnable processes, is the fundamnetal decision the scheduler must take.

Time slice: Linux uses a tick to allocate processor time to individual processes. There are several different hardware timers that the kernel can access. One is loaded at boot time with values to interrupt the system every tick. The kernel uses the CPU clock speed given as $\mathrm{HZ}$ in the kernel to set the length of a single tick to approximately 2.5 nanoseconds [2]. Consequently, every 2.5 nanoseconds, the system takes over, decrements the amount of ticks that the current process has, and returns the CPU to the process for another tick. If the process running is out of CPU time, the scheduler is invoked. If there are more processes in the run-queue that have been allocated CPU time, the scheduler will find the best one and switch to it. The best process is selected using the task_timeslice() macro defined in sched.c. This task_timeslice() macro calculates a weight for the process using bonus which is calculated from nice values etc.. The highest weight is the best process to schedule [14] [2].

Process Descriptor: The process descriptor [13] is defined in sched.h and holds all information about a process. The amount of ticks of CPU time that a process receives before another process is scheduled is stored in the process descriptor. When all processes in the run-queue have exhausted their CPU time, the scheduler recalculates the amount of CPU time for each process using the macro task_timeslice(). The task_timeslice () uses the process' nice value in the process descriptor to determine the number of ticks the process gets. Here a process is referred to as $p$. Variables in the process descriptor are referred to as $p \rightarrow$ variable. For example $p \rightarrow$ nice refers to the nice value of process $p$.

Context switching: The context of a process [3] consists of the contents of its (user) address space and the contents of hardware registers and kernel data structures that relate to the process. In multi-processing environments, a context switch (or process switch or task switch) is when one process is suspended from execution, its context is recorded and another process starts its execution in the CPU. 


\section{IMPLEMENTATION}

In this section, we briefly desribe about kernel modifications and process charcterization. The kernel modifications subsection shows the kernel data structures modified for finding STS. The process characterization section shows, how to find the static and dynamic characteristics of a process.

\section{A. Kernel modifications}

Our idea of kernel modifications is similar to that of [12], but the difference is that we describe the modifications to $\mathrm{O}(1)$ scheduler of Linux Kernel 2.4.20-8.

1) Process descriptor: The Linux process descriptor is defined in sched.h as a structure called task_struct. More than one process can request a larger amount of processor time and the amount of extra time to be given is specific to the process. Therefore the extra time a process gets is stored in the process descriptor. A single integer field, special_time_slice is added to the process descriptor and is sufficient to store the number of ticks that a CPU-bound process should receive. This single variable can also be used to distinguish between CPU-bound process from the other processes in the system. If $p \rightarrow$ special_time_slice is less than 0 then this process should be allocated the standard amount of ticks defined by the macro task_timeslice() in sched.c. If $p \rightarrow$ special_time_slice is greater than 0 then the process is given special_time_slice ticks of processing time instead of using the standard macro, task_timeslice(). This requires that when processes are created, the special_time_slice variable must be initialized to -1 in fork.c. The next sections describe how the modified scheduler uses the special_time_slice variable in the process descriptor.

2) System calls: Two system calls have been added to the kernel for controlling CPU-bound processes. One system call is necessary to allocate more processor time for a single process. This system call performs two simple tasks. First, the special_time_slice variable is set to the number of ticks requested by the process via an argument to the system call. Second, the time_slice variable of the process descriptor is set to the value passed to the system call. The process then returns to run on the processor with an increased amount of processor time and special handling from the scheduler. Therefore the process gets special_time_slice ticks of processor time, each time the scheduler recalculates the process time_slices.

The second system call is used to return a process to the normal state in which $p \rightarrow$ time_slice is calculated using task_timeslice() and $p \rightarrow$ special_time_slice is less than 0 . The next time the scheduler runs the time_slice is set to task_timeslice $(p)$, which is the standard method of assigning $p \rightarrow$ time_slice.

3) Modifications to $O(1)$ scheduler data structures: The scheduler_tick() function of sched.c has been modified to handle any process with special_time_slice $>0$ differently from other processes.If this value is exhausted (decremented to $0)$ then the scheduler gives the processor to the next process. The run-queue is a circular doubly linked list of process descriptors in the TASK_RUNNING state. The first thing that the new scheduler does is check whether process currently running has been granted more processor time $(\mathrm{p} \rightarrow$ special_time_slice $>0$ ). If this is true, and the process still has some CPU time left $(\mathrm{p} \rightarrow$ time_slice $>0$ ), then the scheduler immediately gives the processor back to the current process. This guarantees that each processor-bound process uses all of its processor time immediately, even if the scheduler is called in the middle of its running. If the CPU-bound process has used all of its CPU time $(\mathrm{p} \rightarrow$ time_slice $>0$ ), then the scheduler continues its normal operation and switch to the next process.

Calculating the goodness [2] for each process remains the same. Since CPU-bound processes have large time_slice values, they are not favored over other processes. This is acceptable since the CPU-bound processes are typically the only things using the processor on a system. The CPUbound process may be last to run, but there should not be much waiting to run in front of them, and from the previous modification these processes is guaranteed to stay on the processor until they have used all of there CPU time.

A slight modification to the recalculation of the time_slice variables checks each process to see if it can be allotted more than task_timeslice $(p)$ ticks of processing time. If the $p \rightarrow$ special_time_slice $>0$, then $p \rightarrow$ time_slice is set to $\mathrm{p} \rightarrow$ special_time_slice. There is a small amount of overhead added to the scheduling algorithm, because each process has to be checked to see if it is a CPU-bound process. All other processes receive the same time_slice value, as they would under normal operation of the scheduler. The rest of the scheduling algorithm remains the same.

\section{B. Process characterization}

This section describes, how we characterized the programs. We studied the execution behaviour of several programs to find out the characteristics that can be used to predict the STS. We have taken representative programs : matrix multiplication, sorting programs, recursive fibnocci number generating programs and random number generator programs. Any Machine learning technique [15] requires fully labeled (categorized) data for training. We categorized our data into classes and trained the classifiers (machine-learning techniques) [15].

The experimental procedure is divided into two phases. In the first phase, we create the data set from the program's run traces and make the data base with the static and dynamic charactersitics of the programs and train it with machine learning techniques. In the second phase, we classify this data according to the fitness function, "STS" by using machinelearning techniques. The trained classifiers are then used on a different data set called as a test data set. Often due to limitations in size of the data, we train classifiers using a leaveone-out technique [16] which is a standard technique.

1) Creating the dataset: To characterize the program execution behaviour, we need to find the static and dynamic characteristics. We used readelf [1] and size commands to get the attributes as shown in Table I. To find STS, a script ran the matrix multiplication program of size $700 \times 700$ multiple times with different values of STS on P4 Linux 
TABLE I

ATTRIBUTES AND THEIR MEANING

\begin{tabular}{|c|c|}
\hline Attribute & Definition \\
\hline Hash & This is symbol hash table size in bytes. \\
\hline Dynamic & This dynamic linking information size in bytes. \\
\hline$\overline{\text { Dynstr }}$ & $\begin{array}{l}\text { This is size ( bytes )of strings needed for } \\
\text { dynamic linking. }\end{array}$ \\
\hline $\mathrm{msh}$ & This is symbol hash table size in bytes. \\
\hline Dynamic & This dynamic linking information size in bytes. \\
\hline Dynstr & $\begin{array}{l}\text { This is size ( bytes )of strings needed for dy- } \\
\text { namic linking, most commonly the strings that } \\
\text { represent the names associated with symbol } \\
\text { table entries. }\end{array}$ \\
\hline Dynsym & $\begin{array}{l}\text { This is size ( bytes) of the dynamic linking } \\
\text { symbol table. }\end{array}$ \\
\hline Got & This is the global offset table size in bytes. \\
\hline Plt & $\begin{array}{l}\text { This is the procedure linkage table size in } \\
\text { bytes. }\end{array}$ \\
\hline RoData & $\begin{array}{l}\text { This is read-only data size (bytes) that typically } \\
\text { contribute to a non-writable segment in the } \\
\text { process image. }\end{array}$ \\
\hline Ryl.Dyn & $\begin{array}{l}\text { This is the size (bytes) of the rellocation infor- } \\
\text { mation size. }\end{array}$ \\
\hline Text & $\begin{array}{l}\text { This is the "text" or "executable instructions", } \\
\text { size (bytes )of a program. }\end{array}$ \\
\hline Data & $\begin{array}{l}\text { This is the size(bytes) of the initialized data } \\
\text { that contribute to the size of the program's } \\
\text { memory image size. }\end{array}$ \\
\hline Bss & This section holds uninitialized data size \\
\hline Total_size & This is total size (bytes) of the program. \\
\hline Program_Name & $\begin{array}{l}\text { This is name of the program and a nominal } \\
\text { attribute. }\end{array}$ \\
\hline$\overline{\text { Input_Size }}$ & $\begin{array}{l}\text { Value of InputSize which depends on the pro- } \\
\text { grams type. }\end{array}$ \\
\hline Input_Type & Type of the Input which is a nominal value. \\
\hline SpecialTimeSlice(STS) & Value of the best time slice. \\
\hline STSclass & Class of the STSs \\
\hline
\end{tabular}

System and selected the STS, which gives minimum TaT. The first 18 (characteristics) attributes are used to predict the target attribute STSclass.

We build the data set of 84 execution instances of five programs: matrix multiplication, quick sort, merge sort, heap sort and a recursive Fibonacci number generator and the Table II shows an example of a training example or an instance. We collected the data like the above for 5 programs with different input sizes and different best STSs. Data of 84 instances of the five programs was collected and made into 11 categories based on the attribute STSclass with each class having an interval of 50 ticks.

2) Training and Testing methodology: We performed two types of tests on the training examples with all the learners described in the section, "Overview of machine learning algorithms", on the data sets collected in the first phase. The tests are:

- Use Training Set [9]: The classifier is evaluated on how well it predicts the class of the instance it was trained on.

- Cross-Validation [9]: The classifier is evaluated by crossvalidation, using the number of folds that are entered in the Folds text field (Weka). Recognition accuracy was tested via cross-validation. In this test, the training examples are divided into 10 parts and the classifier classifies by taking one part as a test set and other 9 parts as training set. Likewise, we continue for all parts.
TABLE II

THE INSTANCE

\begin{tabular}{|l|r|}
\hline Attribute & Value \\
\hline Hash & 44 \\
\hline Dynamic & 12 \\
\hline Dynstr & 32 \\
\hline Dynsym & 56 \\
\hline Got & 8 \\
\hline Plt & 32 \\
\hline RoData & 208 \\
\hline Ryl.Dyn & 48 \\
\hline Text & 2400 \\
\hline Data & 5200 \\
\hline Bss & 12000 \\
\hline Total_size & 18000 \\
\hline Program_Name & mm \\
\hline Input_Size & 800 \\
\hline Input_Type & i1 input_type1 \\
\hline SpecialTimeSlice (STS) & 110 \\
\hline STSclass & t2 (101 ticks-149 ticks) \\
\hline
\end{tabular}

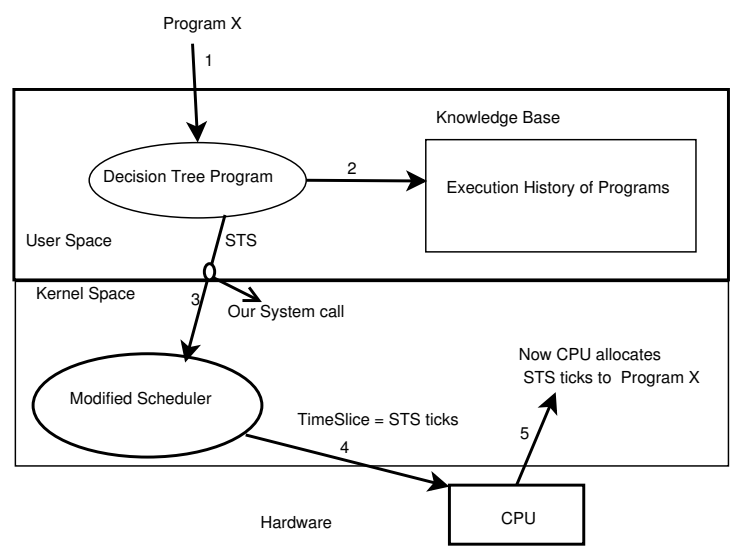

Fig. 1. The design of modified scheduling process.

The results of these tests explained in the next section "Evaluation and Results".

3) Extracting the best attributes: Attributes selection ${ }^{1}$ [11] involves searching through all possible combinations of attributes in the data to find which subset of attributes works best for predicting the program execution behavior. Here our goal is to predict the target attribute. To do this, two objects must be set up ; "an attribute evaluator "and "a search method". The evaluator determines what method is used to assign a value (weight or worth) to each subset of attributes. Here, we used Exhaustive and Genetic search methods, and Co-rrelation based feature selection (CfsSubsetval) [9] evaluation method. A detailed description of feature selection is presented in [11].

\section{The design of modified scheduling process}

The Figure 1 shows the design of modified scheduling process and the steps to minimize TaT of a program. The steps to minimize TaT of a program are as shown in the Figure 1 with numbers from 1 to 5 .

1) The program ' $X$ ' is given to $C 4.5$ decision tree as an input.

\footnotetext{
${ }^{1}$ Extracting the best attributes is nothing but feature selection
} 
2) The decision tree will classify ' $X$ ' and output the STS.

3) We send this STS information to modified scheduler through a system call.

4) The scheduler instructs the CPU such that CPU allocates STS ticks to ' $X$ '.

5) The CPU allocates STS ticks to ' $X$ ' and it will run with minimum TaT.

\section{Overall method}

Our method is explained as follows :

1) Run the programs with different special_time_slices with modified $\mathrm{O}(1)$ scheduler and find STS (best special_time_slice) which gives minimum turn-around-time (TaT).

2) Build the knowledge base of static and dynamic characteristics of the programs from the run traces obtained in step 1 and train them with the $\mathrm{C} 4.5$ decision tree algorithm.

3) If a new program comes, classify it and run the program with this predicted STS.

4) If the new program instance is not in the knowledgebase, go to step 1 .

\section{E. Experimental environment}

For this work, we used GNU/Linux 2.4.20-8 operating system, 1.6GHz Intel P4 processor, 128MB RAM main memory, 512KB Cache memory, $V i$ editor and GNU gcc compiler and so on. WEKA(Waikato Environment for Knowledge Analysis), an open source machine-learning tool was used to find the most suitable ML method to characterize our programs.

\section{EXPERIMENTS AND RESULTS}

We explain the experimentation process by taking the matrix multiplication program as an example.

\section{A. Effectiveness of STS}

A script ran the matrix multiplication program of size 700 $\mathrm{x} 700$, multiple times with different values of STS on P4 Linux System. The table III shows how the turn-aroundtime changed as the CPU burst time (STS) of the process changed. From the table III, STS (or Best special_time_slice)

TABLE III

EFFECT OF SPECIAL_TIME_SLICE ON TURN-AROUND-TIME(TAT).

\begin{tabular}{|l|l|}
\hline Average TaT(secs) & special_time_slice (no.of ticks) \\
\hline 16.372123 & 100 \\
\hline 16.900567 & 150 \\
\hline 15.863590 & 200 \\
\hline$\ldots .$. & $\ldots .$. \\
\hline 15.880436 & 1200 \\
\hline$\ldots$ & $\ldots$ \\
\hline
\end{tabular}

is 200 and corresponding TaT $=15.863590$ sec. Standard TaT (with unmodified scheduler) is $16.466 \mathrm{sec}$. The microseconds saved per second $=(16.466880-15.863590) / 16$ $=60320$ micro-seconds. About 60320 micro-seconds saved per second.file:///usr/share/doc/HTML/index.html Therefore on $1.6 \mathrm{GHz}$ Intel $\mathrm{P} 4$ machine, we can save $1600 \mathrm{MHz}=16 \mathrm{x}$ $10^{8}$ clocks/second $=\left[16 \times 10^{8} \times\right.$ no.of pipelines $\left.\times(60320)\right]$ low-level operations, with special_time_slice $($ STS $)=200$.

\section{B. Selecting the best machine Learning technique}

We used WEKA for training and testing the data set of 84 instances of those five programs to find a good ML technique [8]. The table IV shows the best machine learning technique particularly for our data set. From the table IV, C4.5 is the

TABLE IV

Machine LeARning TeChNiQUes ANd Prediction ACCURACIES on 84 INSTANCES.

\begin{tabular}{|l|l|l|}
\hline ML Technique & $\begin{array}{l}\% \text { Classfication with } \\
\text { Cross Validation Test }\end{array}$ & $\begin{array}{l}\% \text { Classification with } \\
\text { Use Training Set Test }\end{array}$ \\
\hline C4.5(J48) & $91.1667 \%$ & $94.0476 \%$ \\
\hline IBK & $89.5432 \%$ & $93.6480 \%$ \\
\hline
\end{tabular}

best technique ${ }^{2}$ to predict the STS [8].

C. Finding the best attributes which can characterize STS class

We applied, exaustive search with $C f$ s Subset [11] evaluation method on the training set and find the best attributes, which can characterize the STS Class. The Table V shows the best attributes.

TABLE V

The Best AtTributes.

\begin{tabular}{|l|r|}
\hline Attribute & Its Rank \\
\hline InputSize & 1 \\
\hline ProgramSize & 2 \\
\hline BSS & 3 \\
\hline RoData & 4 \\
\hline Text & 5 \\
\hline InputType & 6 \\
\hline
\end{tabular}

D. Effectiveness of our method in reducing the Turn-aroundtime.

The table VI shows that how our Modified Scheduler ${ }^{3}$ reduces turn-around-time of Matrix Multiplication program with different input sizes. In table VI, MS is Modified Scheduler and UMS is Unmodified Scheduler.

Influence of STS on a heavy load system : We ran the matrix multiplication programs as a batch on a heavy load system to test the effectiveness of our modified scheduler. There are 10 programs in a batch. The table VII shows the results. We ran four programs which have operations like opening several files, writing large number of random numbers in them and closing them to make the system as a heavy load system. We monitored the load-average using "top" command.

\footnotetext{
${ }^{2}$ IBK is also a good technique but it is costly in terms of computation as compared to $\mathrm{C} 4.5$

${ }^{3}$ UnModified Scheduler is $\mathrm{O}(1)$ Scheduler of Linux Kernel 2.4.20-8 and Modified scheduler is modified O(1) Scheduler of Linux Kernel 2.4.20-8.
} 
TABLE VI

THE COMPARISION OF UNMODIFIEd SCHEDULER AND MODIFIED Scheduler of Matrix Multiplication Programs.

\begin{tabular}{|l|l|l|l|}
\hline Matrix Sizes & $\begin{array}{l}\text { TaT with UnModi- } \\
\text { fied Scheduler }\end{array}$ & $\begin{array}{l}\text { TaT with Mod- } \\
\text { ified Scheduler }\end{array}$ & $\begin{array}{l}\text { Reduction in } \\
\text { TaT }\end{array}$ \\
\hline $700 \times 700$ & 16466 millisecs & 15864 millisecs & 602 millisecs \\
\hline $750 \times 750$ & 19568 millisecs & 19228 millisecs & 340 millisecs \\
\hline $800 \times 800$ & 23820 millisecs & 23190 millisecs & 630 millisecs \\
\hline $850 \times 850$ & 28789 millisecs & 28112 millisecs & 677 millisecs \\
\hline $900 \times 900$ & 33540 millisecs & 32029 millisecs & 1511 millisecs \\
\hline $950 \times 950$ & 39997 millisecs & 39388 millisecs & 609 millisecs \\
\hline $1000 \times 1000$ & 47370 millisec & 46110 millisecs & 1260 millisecs \\
\hline $1050 \times 1050$ & 54912 millisecs & 54204 millisecs & 708 millisecs \\
\hline $1100 \times 1100$ & 63782 millisecs & 61580 millisecs & 2200 millisecs \\
\hline $1150 \times 1150$ & 72704 millisecs & 70264 millisecs & 2440 millisecs \\
\hline $1200 \times 1200$ & 85776 millisecs & 80654 millisecs & 5122 millises \\
\hline
\end{tabular}

TABLE VII

COMPARISON OF MODIFIEd SCHEDULER AND UNMODIFIEd SCHEDULER ON A HEAVY LOAD SYSTEM.

\begin{tabular}{|l|l|l|l|l|l|}
\hline Matrix Sizes & $\begin{array}{l}\text { Batch } \\
\text { Size }\end{array}$ & $\begin{array}{l}\text { load- } \\
\text { average }\end{array}$ & $\begin{array}{l}\text { TaT } \\
\text { with } \\
\text { UMS }\end{array}$ & $\begin{array}{l}\text { TaT } \\
\text { with } \\
\text { MS }\end{array}$ & $\begin{array}{l}\text { Reduction } \\
\text { in TaT }\end{array}$ \\
\hline $800 \times 800$ & 10 & 7.38 & $\begin{array}{l}165.57 \\
\text { secs }\end{array}$ & $\begin{array}{l}163.23 \\
\text { secs }\end{array}$ & $\begin{array}{l}2.34 \\
\text { secs }\end{array}$ \\
\hline $900 \times 900$ & 10 & 10.34 & $\begin{array}{l}469.23 \\
\text { secs }\end{array}$ & $\begin{array}{l}463.86 \\
\text { secs }\end{array}$ & $\begin{array}{l}5.37 \\
\text { secs }\end{array}$ \\
\hline
\end{tabular}

Decision tree overhead: We implemented C4.5 decision tree for training and testing and its running time is around 220 milliseconds. The overhead of this is around $4 \%$ when compared to the reduction in turn-around time in case of matrix multiplication program of size $1200 \times 1200$. So we can neglect the overhead of decision tree.

Limitations of our method.: The characteristics of the programs and the gain in the turn-around-time depends on the architecture and operating system. Our modified scheduler is not designed with any security features that would prevent a user from writing their own process and requesting INT_MAX processor time via system call which is described in the Section V-A.2. Setting $p->$ special_time_slice to INT_MAX could significantly slow down a system.

\section{CONCLUSIONS}

By considering the static and dynamic characteristics of a program, we can schedule it using modified scheduler such that the turn-around-time of it is minimized. We show that machine learning algorithms are very efficient in the process characterization process. The C4.5 decision tree algorithm achieved good prediction $(91 \%-94 \%)$, which indicates that when suitable attributes are used, a certain amount of predictability does exist for known programs. Our experiments show that $1.4 \%$ to $5.8 \%$ reduction in turn-aroundtime is possible and this reduction rate slowly increases with the input size of the program. From our experiments, we find the best features : input size, program size, bss, text, rodata and input type of a program that can characterize its execution behavior. We conclude that our technique can improve the scheduling performance in a single system.
Future work: Our future work will include extending our technique to parallel programs, more comprehensive study of high performance application characteristics is being conducted on an 8-node Linux cluster, and will also include to add our idea of predictability to Portable Batch Scheduler of Linux Cluster.

\section{ACKNOWLEDGMENT}

We would like to thank Sasi Kanth Ala for giving valuable advice through out of our work.

\section{REFERENCES}

[1] Hongjiu Lu, ELF: From The Programmer's Perspective, Technical Report, NYNEX Science and Technology, 500 Westchester Avenue, White Plains, NY 10604, USA, May, 1995.

[2] Aivazian, Tigran, Linux Kernel 2.4 Internals, The Linux Documentation Project, August, 2002.

[3] Maurice Bach, The design of the Unix operating system, Pearson Education Asia, pp:159-170, 2002.

[4] Fredrik Vraalsen, "Performance Contracts: Predicting and monitoring grid application behavior", In Proceedings of the 2nd Internationsl Workshop on Grid computing, November, 2001.

[5] Richard Gibbons, A Historical Application Profiler for Use by Parallel Schedulers, Lecture Notes on Computer Science, Volume : 1297, pp: 58-75, 1997.

[6] Hyok-Sung Choi and Hee-Chul Yun, Context Switching and IPC Performance Comparison between uClinux and Linux on the ARM9 based Processor, Linux in embedded applications, Jan., 2005. http://www.linuxdevices.com/articles/AT2598317046.html.

[7] Warren Smith, Valerie Taylor, Ian Foster, "Predicting Application RunTimes Using HistoricalInformation", Job Scheduling Strategies for Parallel Processing, IPPS/SPDP'98 Workshop, March, 1998.

[8] Kishore Kumar. P and Atul Negi, Characterizing Process Execution Behaviour Using Machine Learning Techniques, In DpROM WorkShop Proceedings, HiPC 2004 International Conference, December, 2004.

[9] Garner, S .R., "WEKA: The Waikato Environment for Knowledge Analysis", In Proc. of the NewZealand Computer Science Research Students Conference, pp : 57-64, 1995.

[10] Surkanya Suranauwarat, Hide Taniguchi, "The Design, Implementation and Initial Evaluation of An Advanced Knowledge -based Process Scheduler", ACM SIGOPS Operating Systems Review, volume: 35, pp: 61-81, October, 2001.

[11] Mark A. Hall, Co-rrelation based feature selection for Machine Learning, Master Thesis, Department of Computer Science, University of Waikato, pp: 7-9, 12-14, April, 1999.

[12] Andrew Marks, A Dynamically Adaptive CPU Scheduler, Master Thesis, Department of Computer Science, Santa Clara University, pp :59, June, 2003.

[13] Robert Love, Linux Kernel Development, 1st ed, The Pearson Education, 2004.

[14] Danie P. Bovet, Marc, Understanding the Linux Kernel, 2nd ed, O' Reilly and Associates, Dec., 2002.

[15] Tom Mitchell, Machine Learning, 1st ed, pp: 52-75, 154-183, 230-244, The Mc-Graw Hill Company.Inc.International Edition, 1997.

[16] O. Duda, P. E.Hart, Pattern Classification, Wiley, NewYork,1973.

Atul Negi works as a reader in the Department of Computer and Informations Sciences, University of Hyderabad, Hyderabad. He is presently the IEEE Computer Society Chapter Chair for IEEE Hyderabad Section. His interests are in applications of Machine Learning, Pattern Recognition and Document Image Analysis.

Kishore Kumar P. is a Masters student in the Department of Computer and Informations Sciences, University of Hyderabad, Hyderabad. His interests are in Operating Systems and Cluster computing. 